\title{
Construção de saberes interdisciplinares em redes: limites e possibilidades de um grupo de pesquisa
}

\author{
The construction of interdisciplinary knowledge in networks: limitations and possibilities of a research group
}

Construcción de conocimientos interdisciplinarios en redes: límites y posibilidades de un grupo de investigación

Maria das Graças Carvalho Ferriani ${ }^{1}$, Marta Angélica lossi Silva ${ }^{2}$, Lygia Maria Pereira da Silva ${ }^{3}$, Liliana Scatena ${ }^{4}$, Maria Aparecida Beserra ${ }^{5}$

\footnotetext{
${ }^{1}$ Enfermeira, Doutora em Enfermagem. Professora Titular da Escola de Enfermagem de Ribeirão Preto da Universidade de São Paulo (EERP/USP). Ribeirão Preto, SP, Brasil. E-mail: caroline@eerp.usp.br.

${ }^{2}$ Enfermeira, Doutora em Enfermagem em Saúde Pública. Professora Doutora da EERP/USP. Ribeirão Preto, SP, Brasil. E-mail: maiossi@eerp.usp.br.

${ }^{3}$ Enfermeira, Doutora em Enfermagem. Professora Assistente da Universidade de Pernambuco. Recife, PE, Brasil. E-mail: lygiapera@yahoo.com.br.

${ }^{4}$ Enfermeira. Ribeirão Preto, SP, Brasil. E-mail: liliana scatena@hotmail.com.

${ }^{5}$ Enfermeira, Mestre em Enfermagem. Recife, PE, Brasil. E-mail: mcidabeserra@ig.com.br.
}

\section{RESUMO}

O objetivo deste estudo foi conhecer como se configuram os vínculos entre o Núcleo de Estudo, Ensino e Pesquisa do Programa de Assistência Primária de Saúde Escolar e as instituições envolvidas na atenção às crianças e aos adolescentes, assim como nas atividades de ensino, pesquisa e extensão. Trata-se de estudo descritivo-exploratório, de abordagem qualitativa, que teve como campo o Departamento de Enfermagem Materno-Infantil e Saúde Pública da Escola de Enfermagem de Ribeirão PretoUSP. A coleta de dados foi realizada por meio do mapeamento dos vínculos da rede institucional externa e os sujeitos foram nove membros do núcleo. Constatou-se a fragilidade ou inexistência de articulação com parceiros nos extremos de distância geográfica, sendo os mais próximos outros núcleos da Escola e os mais distantes, as instituições internacionais. $O$ aumento dos vínculos significativos, visando ao fortalecimento da rede, constitui-se em grande desafio para o núcleo.

Descritores: Grupos de Pesquisa; Educação em Enfermagem; Comunicação Interdisciplinar; Rede Social.

\section{ABSTRACT}

The objective of this study was to understand the connections between the Study, Teaching and Research Center of the Primary Healthcare School Program and the institutions involved in the care delivered to children and teenagers, as well as the activities of education, research and outreach. This descriptive-exploratory qualitative study was performed with the Department of Maternal-Child and Public Health Nursing of the Ribeirão Preto College of Nursing, University of São Paulo. Data collection was performed by means of the mapping of the connection of the outer institutional network. The subjects were nine members of the referred center. The closest partners were other centers in the College, and the most distant, geographically, were the international institutions; and connections with the latter were either weak or inexistent. Increasing significant connections, with a purpose to strengthen the network, is a great challenge for the center.

Descriptors: Research Groups; Education, Nursing; Interdisciplinary Communication; Social Networking.

\section{RESUMEN}

El estudio objetivó conocer la configuración de los vínculos entre el Núcleo de Estudio, Enseñanza e Investigación del Programa de Atención Primaria de Salud Escolar y las instituciones involucradas en la atención de niños y adolescentes, así como las actividades de enseñanza, investigación y extensión. Estudio descriptivo, exploratorio, cualitativo, que tuvo como campo de realización al Departamento de Enfermería Materno Infantil y Salud Pública de la Escuela de Enfermería de Ribeirão Preto-USP. Datos recolectados mediante mapeo de los vínculos de la red institucional externa. Los sujetos fueron nueve miembros del núcleo. Se constató la debilidad o inexistencia de articulación con asociados en los extremos de distancia geográfica, constituyendo los más próximos otros núcleos de la Escuela, y los más distantes, las instituciones internacionales. El aumento de los vínculos significativos, apuntando al fortalecimiento de la red, se configura como gran desafío para el núcleo.

Descriptores: Grupos de Investigación; Educación en Enfermería; Comunicación Interdisciplinaria, Red Social 


\section{INTRODUÇÃO}

O avanço e a ampliação do conhecimento em Enfermagem são verificados frente à busca pela articulação do conhecimento, com vistas ao desenvolvimento global da sociedade. É notório o avanço do conhecimento de Enfermagem no Brasil pelos estudos apresentados na literatura advindos dos grupos de pesquisa ${ }^{(1-8)}$. Grupos de pesquisa em Enfermagem estão cadastrados no CNPq desde a década de 1970, percebendo-se um crescimento significativo dos mesmos na área da Enfermagem, o incremento da produção científica e a qualificação dos integrantes, que ocorre de acordo com a evolução da pós-graduação no Brasil. Isso caracteriza-se pelo incentivo e direcionamento da produção do conhecimento das linhas de pesquisa, ou seja, a sistematização da produção científica(2).

O grupo de pesquisa é definido como um conjunto de indivíduos organizados hierarquicamente em torno de uma ou, eventualmente, duas lideranças: o fundamento organizador dessa hierarquia é a experiência, o destaque e a liderança no terreno científico ou tecnológico; profissionais estão envolvidos permanentemente com atividades de pesquisa, cujo trabalho se organiza em torno de linhas comuns de investigação; em algum grau, compartilham instalações e equipamentos ${ }^{(9)}$. Reitera-se que as atividades de pesquisa contribuem também, para além na formação na pós-graduação, para a formação dos acadêmicos na iniciação científica que, no desenvolvimento de todas as etapas operacionais da pesquisa, tem construído o conhecimento nos quatro pilares da educação: aprender a conhecer, aprender a fazer, aprender a ser e aprender a viver juntos. Esse processo tem preparado acadêmicos de graduação para continuarem na carreira acadêmica por meio do ingresso em cursos de pós-graduação, lato-sensu e/ou strictosensu e mesmo a não se distanciarem do conhecimento científico atualizado quando estes passam a atuar na rede de saúde ${ }^{(10)}$.

Considerada a inter-relação e interação das disciplinas, por meio da interdisciplinaridade, busca-se atingir um objetivo comum e a unificação conceitual dos métodos e estruturas, nos quais as potencialidades das disciplinas são exploradas e ampliadas no contexto dos grupos de pesquisa. O agir e o pensar interdisciplinar, por meio do qual se busca o diálogo com outra forma de conhecimento e outras metodologias, objetiva e potencializa a construção de novos conhecimentos. A interdisciplinaridade se apresenta como resposta à diversidade, à complexidade e à dinâmica do mundo atual(11). A interdisciplinaridade é também a ruptura das fronteiras disciplinares em direção, fundamentalmente, a novos sítios de conhecimento. Refere-se à troca de informações de conhecimento entre disciplinas, à utilização dos métodos de uma disciplina por outras. "A interdisciplinaridade possibilita não só a fecunda interlocução entre diferentes áreas do conhecimento, como também constitui uma estratégia importante para que elas não se estreitem e nem se cristalizem no interior de seus respectivos domínios"(12).

A interdisciplinaridade deve ser entendida como método, caracterizado pela intensidade das trocas entre especialistas e pela interação real das disciplinas dentro de um mesmo projeto, através de relações de interdependência e de conexões recíprocas, o que não deve ser confundido com simples trocas de informações ${ }^{(13)}$.

Com a fundamentação nos conceitos apresentados, teve-se como objeto deste estudo os núcleos de pesquisa da Escola de Enfermagem de Ribeirão Preto da Universidade de São Paulo (EERP/USP), cadastrados no diretório do Conselho Nacional de Pesquisa (CNPq). A Escola de Enfermagem conta com 32 grupos de pesquisa, entretanto, o interesse, aqui, é ter como recorte de estudo o Núcleo de Estudo, Ensino e Pesquisa do Programa de Assistência Primária de Saúde Escolar PROASE, cadastrado no diretório do CNPq, desde 1985.

Justifica-se tal opção por se considerar importante estudar e analisar como vem se configurando o grupo no qual uma das autoras atua, desde sua origem, trabalhando com profissionais, pesquisadores, alunos de graduação e pós-graduação das diversas áreas do conhecimento, com vistas a atender as necessidades da criança em idade escolar e do adolescente e também de comparar a atuação do grupo com o cenário brasileiro. As temáticas, atualmente investigadas pelos pesquisadores do grupo, são consideradas pela maioria dos autores como grandes problemas para a saúde pública, a saber: adolescência, violência doméstica e institucional, educação e promoção da saúde, que se constituem, também, em grande desafio para as políticas públicas.

Desse modo, levantaram-se as seguintes questões: quais os vínculos estabelecidos pelo PROASE com a 
graduação, pós-graduação, grupos de pesquisa da EERP/USP, Secretarias Municipais de Saúde, Educação e Cidadania, parceiros nacionais e internacionais, pesquisadores e órgãos de fomento, no que concerne às atividades de ensino, pesquisa e extensão? Quais os limites do trabalho do PROASE e as estratégias utilizadas para o fortalecimento da pesquisa? Quais os limites e as possibilidades que o Núcleo considera para trabalhar com saberes interdisciplinares em redes?

Para responder às questões de pesquisa, estabeleceu-se como objetivo do presente estudo: conhecer como se configuram os vínculos entre o PROASE e as instituições envolvidas na atenção às crianças e adolescentes, assim com nas atividades de ensino, pesquisa e extensão.

\section{METODOLOGIA}

Trata-se de estudo descritivo exploratório(14), de abordagem qualitativa, que é considerada como aquela que "se aplica ao estudo da história das relações, das representações, das crenças, das percepções e das opiniões, produtos das interpretações que os humanos fazem a respeito de como vivem, constroem seus artefatos e a si mesmos, sentem e pensam (...) além de permitir desvelar processos sociais ainda pouco conhecidos referentes a grupos particulares, propiciar a construção de novas abordagens, revisão e criação de novos conceitos e categorias durante a investigação." (14)

Elegeu-se como campo de estudo o Departamento de Enfermagem Materno-Infantil e Saúde Pública da EERPUSP, onde o PROASE tem sua sede principal. O Núcleo conta com um Laboratório Óptico, instalado no espaço físico da EERP, desde 1990. A área física do Laboratório corresponde a $42 \mathrm{~m}^{2}$, contemplando três salas, sendo uma de atendimento à comunidade, outra para a guarda do material e uma sala destinada aos pesquisadores do grupo (bolsistas, graduandos, pós-graduandos, apoio técnico), com armários, computadores e impressoras.

Cabe ressaltar que as investigações do PROASE desenvolvem-se em diferentes campos nacionais e internacionais, a saber: nacionais - Ribeirão Preto/SP, Jardinópolis/SP, SP, Alfenas/MG, Uberaba/MG, Campinas/SP, Guarulhos/SP, Recife/PE, Feira de Santana/BA, Goiânia/GO, Rondônia/RO; internacionais México, Argentina, Chile, Venezuela e Equador. Como critérios de inclusão, adotamos todos sujeitos do grupo do PROASE presentes na oficina.

A presente pesquisa teve como sujeitos 0 coordenador e vice-coordenador do grupo (2), pósdoutorando (1), doutorandos (2), mestrando (1), aluno de iniciação (1) e pesquisadores permanentes (2).

O estudo atendeu os princípios éticos da pesquisa com seres humanos, estabelecidos pelo Conselho Nacional de Saúde, por meio da Resolução 196/96. Todos os sujeitos foram informados e esclarecidos sobre o objetivo da pesquisa e assinaram o Termo de Consentimento Livre e Esclarecido (TCLE). A pesquisa foi aprovada pelo Comitê de Ética e Pesquisa da Escola de Enfermagem de Ribeirão Preto/USP - protocolo $n^{\circ}$ $1345 / 2011$.

Como instrumento de coleta de dados, foi utilizado o mapeamento dos vínculos da rede institucional externa ao PROASE, dentro e fora da EERP/USP. Trata-se de metodologia importante para se verificar como os vínculos das instituições são estabelecidos no trabalho cotidiano, permitindo visualizar processos que necessitam ser implementados, fortalecidos e criados ${ }^{(15)}$. Utilizou-se o mapa de redes institucionais, inspirado no mapa mínimo da Rede Pessoal Social(16). Vários autores têm trabalhado sob essa perspectiva, em diferentes áreas, e encontram forte evidência entre a rede social, formada em torno dos indivíduos e das instituições, e a qualidade de vida e de trabalho(15-17).

Trabalhos fragmentados colocam pessoas e instituições como também a sociedade em situação de vulnerabilidade ${ }^{(11,17)}$. Ancoradas nessa lógica, optou-se pela adoção desse instrumento no presente estudo, com base no paradigma de redes, com vistas a diagnosticar como o PROASE vem interagindo com os setores que são importantes para o desenvolvimento das pesquisas, dentre outros elementos.

O mapa é representado por um círculo, dentro do qual se constituem quadrantes, representativos dos diversos setores e instituições, aos quais se liga a instituição estudada. Em seguida, são traçadas linhas do centro (instituição estudada) para os quadrantes (instituições às quais a instituição estudada se vincula) para indicar, por meio da cor e da distância dessa linha, a qualidade dos vínculos existentes entre cada setor ou equipe ${ }^{(11)}$. Na representação gráfica com linhas em cores diferentes, cada cor exprime vínculos: significativos (cor 
verde), vínculos fragilizados (cor amarela) e vínculos inexistentes ou rompidos (cor vermelha). Em relação à distância ${ }^{(18)}$, quanto mais próximo do centro mais forte é o vínculo.

O mapa foi preenchido durante uma oficina realizada com o grupo de pesquisa e alunos matriculados na disciplina de pós-graduação ERM5782-Interpretação de Dados Qualitativos, ministrada pela coordenadora do PROASE. A oficina foi realizada por uma docente convidada, experiente na aplicação e construção desse tipo de mapa, que realizou a coleta de dados com os membros do PROASE, presentes na atividade. Inicialmente, a docente ministrou aula expositivodialogada sobre redes, um novo paradigma que vem sendo utilizado pelos profissionais e pesquisadores da área e a importância dos vínculos entre os setores e instituições; orientação sobre o preenchimento do mapa mínimo, enfatizando que o mesmo se constitui em um diagnóstico. Tal abordagem permitiu aos participantes a visualização dos vínculos institucionais externos do PROASE, estabelecidos com diferentes setores e instituições.

O esquema para a construção do mapa foi previamente desenhado em papel tamanho 0,66x0,98cm, para a boa visualização em aula, contendo, nos respectivos quadrantes, os setores e instituições com os quais o PROASE precisa se vincular para a realização do trabalho. Após a aula sobre redes, foi realizado o preenchimento do mapa, atividade precedida de explicação sumária, buscando-se instrumentalizar os sujeitos participantes. Com o uso de fita adesiva foram colocados os esquemas impressos em uma das paredes da sala, nos quais a facilitadora (docente) preencheu os mapas de acordo com as indicações dos membros.

Após a oficina, foi aplicado um instrumento para complementação das informações, colhidas no preenchimento do mapa ${ }^{(17)}$, visando problematizar as informações fornecidas pelo grupo. As questões foram: se o núcleo de estudos se relaciona bem com cada setor ou instituição apontada no mapa, como é a relação e quais os pontos fortes e os pontos fracos. Outra questão foi se o Núcleo precisa mudar a relação que mantém com as instituições. A síntese dessa discussão foi anotada em diário por um pesquisador colaborador

Foi entregue, posteriormente, aos membros participantes do grupo de estudo uma cópia impressa do mapa preenchido, tamanho A4, para que verificassem os vínculos institucionais do PROASE, informados na ocasião da oficina, permitindo, assim, a visualização do produto elaborado, sua concordância e outras sugestões.

A análise dos dados se iniciou ainda durante a coleta, estando presente em vários estágios da investigação, quando, ainda que parcialmente, foram analisados os achados no campo do estudo(19). Ao término da coleta, a análise tornou-se mais sistemática e mais formal. Os resultados foram descritos e contextualizados com a literatura científica sobre a temática.

\section{RESULTADOS}

Núcleo de Estudos, Ensino e Pesquisa do Programa de Assistência Primária de Saúde Escolar - PROASE, do Departamento de Enfermagem Materno-Infantil e Saúde Pública da EERP-USP

Os vínculos do PROASE com as instituições que atuam na atenção à criança e ao adolescente, assim como sua relação com o ensino, pesquisa e extensão, permitiram a construção de um esquema gráfico, Figura 1, com a configuração. 
Figura 1: Mapa mínimo dos vínculos institucionais do PROASE - Escola de Enfermagem. Ribeirão Preto, SP, 2011.

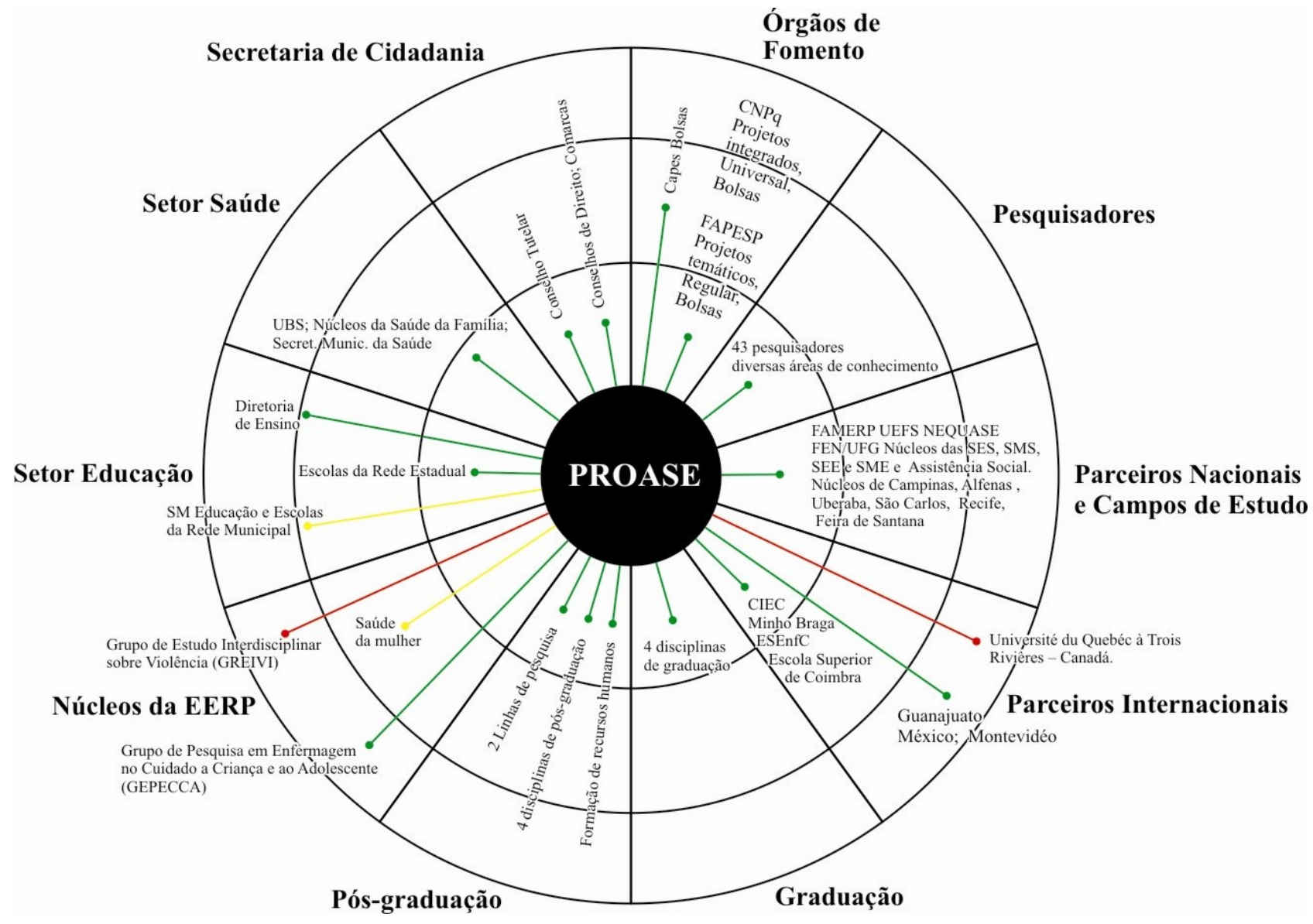

A rede construída pelos sujeitos, tendo como centro o PROASE, é constituída a partir de vínculos entre a equipe de pesquisadores e diversos setores com instituições internas e externas ao núcleo.

A avaliação do mapa dos vínculos ${ }^{(17)}$ revela que a rede, a partir do PROASE, quanto ao seu tamanho, é mediana, considerando-se o número de instituições que se vinculam ao Núcleo. Quanto à densidade, o mapa revela que os traços predominantes são de vínculos significativos, seguidos pelos vínculos fragilizados e, por último, os vínculos rompidos ou inexistentes.

Desde sua implantação, o PROASE tem contribuído para o ensino de graduação, pós-graduação, desenvolvimento de pesquisas, formação de recursos humanos e fortalecimento de duas linhas de pesquisa:

1) Assistência à criança e ao adolescente, que tem como objetivo estudar a assistência à criança e ao adolescente, em seu processo de crescimento e desenvolvimento e em suas relações com a família, escola, instituições de saúde, comunidade e a inserção da Enfermagem nas diversas modalidades de assistência;
2) Sociedade, Saúde e Enfermagem, que busca estudar a articulação teórico-conceitual da estruturação das sociedades e as correspondentes maneiras de produção da saúde e de processos de desenvolvimento da Enfermagem, enquanto prática social.

O Núcleo, atualmente, conta com 43 integrantes das diversas áreas de conhecimento, com caráter multidisciplinar e multiprofissional; tem se dedicado ao desenvolvimento de investigações relacionadas, sobretudo, às temáticas violência doméstica, violência institucional, redes de apoio, educação e promoção à saúde, com projetos financiados pela Fapesp (Fundação de Amparo à Pesquisa do Estado de São Paulo) e CNPq, possibilitando a ampliação do campo de pesquisa para a família, instituições de saúde e educação, instituições de acolhimento, conselhos tutelares, dentre outros. Possui significativa produção técnico-científica, a qual incorpora também pesquisadores de outras universidades nacionais e internacionais e profissionais de diferentes serviços voltados à atenção à criança e ao adolescente.

A rede composta pelos membros e instituições em torno do PROASE pode ser avaliada como heterogênea, 
característica que representa tendência à abertura ${ }^{(20)}$. A interdisciplinaridade da rede se explica tanto pela complexidade do objeto de estudo, que resulta em necessidades em diversas áreas, quanto pelas disciplinas que fundamentam a temática, o referencial teóricometodológico, como é possível constatar pelos vínculos significativos relacionados aos outros grupos de pesquisa da EERP-USP, na graduação e pós-graduação.

O trabalho do PROASE, desenvolvido na perspectiva interdisciplinar, viabiliza a construção de competências investigativas de forma integrada a diversas áreas (antropologia, sociologia, epidemiologia, psicologia e clínica) e a prática de saúde, possibilitando avanços na qualificação de pesquisadores e produção de conhecimento que contribuem para atenção à criança e ao adolescente em diferentes cenários. A formação de recursos humanos pelo Núcleo pode ser visualizada por meio da produção em pesquisa, apresentada no Gráfico 1.

Gráfico 1: Formação de recursos humanos junto ao PROASE no período de 1993-2011. Ribeirão Preto, SP, 2011.

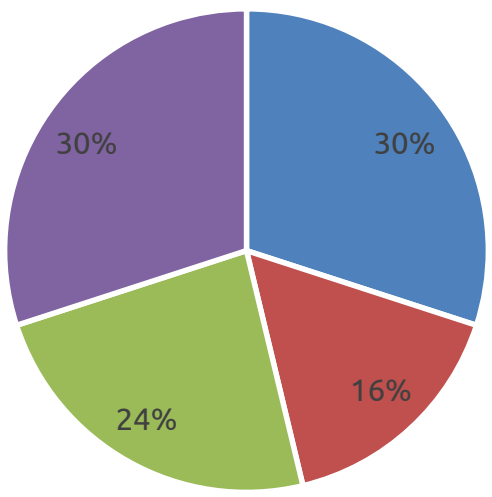

- Iniciação científica

- Monografias

- Dissertações/Mestrado

- Teses/Doutorado

A produção do conhecimento divulgado em periódicos de circulação nacional e internacional, assim como em livros pode ser visualizada no Gráfico 2.

Gráfico 2: Produção do conhecimento do PROASE no período de 1979-2011. Ribeirão Preto, SP, 2011.

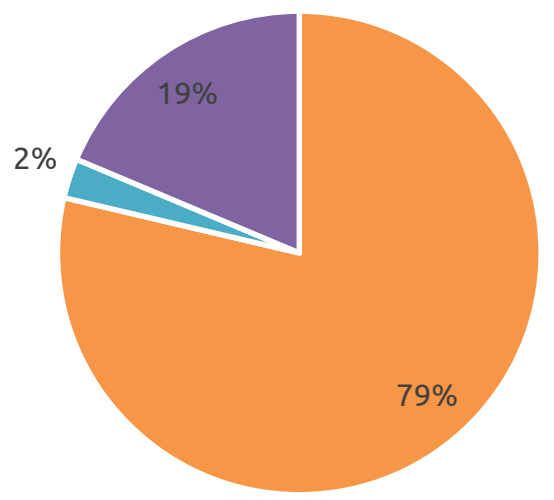

- Artigos publicados

- Livros publicados

- Capítulos de livros

\section{DISCUSSÃO}

O registro da história do PROASE se faz necessário para melhor compreensão do objeto de estudo, não tendo, aqui, a intenção única de fazer história ou registrar fatos do Programa de Saúde Escolar, implantado no município de Ribeirão Preto-SP, em 1985, mas demarcar a 
trajetória do Núcleo, cuja gênese se deu a partir de um trabalho de extensão, que contemplou 92 escolas da Rede Municipal e Estadual de Ensino, com equipe multidisciplinar e se transformou em um Núcleo de Pesquisa.

O trabalho foi iniciado na tentativa de se investigar as razões do descaso pela saúde do escolar em nosso país, não obstante a existência de leis que a regulamentam. A inquietação para o trabalho adveio de inúmeras observações realizadas nessa área. O trabalho realizado há alguns anos permitiu a visualização dos problemas existentes de marginalização do escolar, adolescente em situação de vulnerabilidade social e, ao longo desse processo, buscaram-se articulações dentro do sistema saúde/educação, objetivando atenção mais efetiva à criança e ao adolescente.

Acredita-se ter desenvolvido, na convivência diária com os escolares, as famílias e com as próprias escolas, visão bastante abrangente dos problemas que envolvem os sujeitos, somando-se os dados apresentados na literatura que já vinham sendo pesquisados. Impõe-se, neste momento, registrar como se desenvolveu, paralelamente ao trabalho de extensão à comunidade escolar, a pesquisa pelo Núcleo PROASE.

O objetivo do Núcleo é promover a construção do conhecimento no campo da Educação e Saúde da Criança e do Adolescente, por meio da formação e qualificação de pesquisadores e recursos humanos com ênfase na interdisciplinaridade, multiprofissionalidade e intersetorialidade, ensino de graduação, pós-graduação e trabalhos de extensão à comunidade.

Neste sentido é importante atentar e responder a perspectiva de se interligar três pilares centrais da educação e da saúde, quais sejam: a integração universidade e sociedade; a constituição e a organização de uma equipe multiprofissional para trabalhar em saúde escolar; o recorte intelectual do conhecimento da saúde escolar, ampliando seus limites, passando a considerar a saúde além do espaço do biológico e contextualizando a criança na sua integralidade de pessoa, em suas concretas condições de vida ${ }^{(20-21)}$ sendo que o primeiro deles referese à integração universidade e sociedade (21).

Por meio da experiência de ensino de graduação em Enfermagem, e tendo como meta responder as solicitações e expectativas de saúde da população infantil das escolas de ensino de primeiro grau, da cidade de
Ribeirão Preto, conseguiu-se desenvolver todo um trabalho nessa direção, levando a universidade para dentro da escola e, assim, colocando o conhecimento científico a seu serviço. O segundo pilar é a constituição e a organização de equipe multiprofissional para trabalhar em saúde escolar. Dessa forma, além de demonstrar o papel específico do enfermeiro na promoção e assistência à saúde, conseguiu-se aglutinar outros profissionais e, mais, desenvolver, de fato, trabalho em equipe, com participação efetiva de todos. Construiu-se, assim, o terceiro pilar que se integra aos demais - o recorte intelectual do conhecimento da saúde escolar, ao ampliar seus limites, passando a considerar a saúde além do espaço do biológico e contextualizar a criança na sua integralidade como sujeito, em suas concretas condições de vida.

Diante do resultado da elaboração do mapa, com os vínculos institucionais informados pelos membros do Núcleo, foi possível melhor visualização e compreensão dos três pilares construídos a partir de um programa de extensão(21).

A produção do PROASE, assim como de outros grupos de pesquisa, depende da demanda por recursos financeiros. A articulação dos grupos de pesquisas com políticas institucionais de financiamento se constitui em importante estratégia para o desenvolvimento social, considerando que a pesquisa proporciona o retorno para a sociedade no contexto do incremento científico e tecnológico ${ }^{(2,22)}$.

Em relação à pesquisa, o núcleo submeteu número significativo de projetos aos órgãos de fomento CNPq e Fapesp para captação de recursos, sendo que, em 1997, com o projeto A criança e o adolescente em idade escolar $e$ o adolescente na escola, família e comunidade, Processo n521696/96-8 (CNPq), pode-se considerar o marco, por alavancar o conhecimento dos pesquisadores, conhecimento esse que, ao longo dos anos, tem sido consolidado. Com os resultados obtidos nas diversas pesquisas, foi possível efetuar novas solicitações aos órgãos de fomento e, consequentemente, novas investigações, as quais permitiram responder a diversas e importantes questões e preencher lacunas, em relação a demandas que precisavam ser atendidas.

Paralelamente aos projetos do CNPq, foram demandados projetos temáticos à Fapesp, a exemplo do 
projeto Adolescência e Sexualidade, Processo n०1996/06987-4.

A partir de 2000, emergiu a necessidade de se investigar as questões relacionadas à criança $e$ ao adolescente na escola, família e comunidade, alvos de atos violentos ou vitimas potenciais da violência, e assim foram demandados dois projetos temáticos à Fapesp, já concluídos, além de projetos integrados, projeto universal, projetos de bolsa produtividade e de iniciação científica para o CNPq, também já concluídos.

Está em andamento, um Projeto Universal CNPq (Res)significando a violência na escola: análise e prevenção no contexto escolar, Processo n482842/2010-5; um Projeto de Bolsa Produtividade CNPq, Processo n³02520/2010-4 - As Redes de Apoio Sociais e Afetivas à criança e ao adolescente, vítimas de violência doméstica e institucional; um Projeto Regular - As Redes de Apoio Sociais e Afetivas à criança e ao adolescente vítimas de violência doméstica e institucional, Processo n¹0/517079.

No que concerne à articulação do núcleo com os demais grupos de pesquisa, denota-se que, embora tenha interfaces, decorrentes da temática violência e adolescência, que também tem sido estudada em outros três núcleos, os vínculos identificados são significativos e distantes, com o Grupo de Pesquisa em Enfermagem no Cuidado à Criança e ao Adolescente - GPECCA, vínculos fragilizados com o núcleo Saúde da Mulher, que investiga violência, gravidez na adolescência dentre outros. Com o Grupo de Estudos Interdisciplinares sobre Violência (Greivi), que iniciou suas atividades em 2006, o vínculo é inexistente. As articulações são ainda incipientes e dependem mais de iniciativas individuais das docentes, denotando(12,17-18) a necessidade de mudanças mais profundas, como resultado de uma opção política.

A articulação com o setor Educação se mantém mais próxima, com vínculo significativo com a Diretoria de Ensino e escolas da Rede Estadual, junto às quais vêm sendo realizados projetos de extensão e pesquisa, formação de recursos humanos, grupos de trabalho. No que concerne à Secretaria Municipal de Educação e às Escolas da Rede Municipal, observa-se relação fragilizada, mostrando a dificuldade de articulação para atuação em rede.

Com o setor da saúde, identificou-se vínculo significativo e próximo. Junto a esse setor, o PROASE realiza projetos de extensão, pesquisa e ensino, com a formação de recursos humanos, grupos de trabalho, participação e organização de eventos. Os espaços onde as atividades têm sido realizadas são as unidades básicas de saúde, núcleos da saúde da família e a própria Secretaria Municipal da Saúde.

Os vínculos estabelecidos entre o Núcleo e a Secretaria da Cidadania, especificamente com os Conselhos Tutelares, Conselho de Direito, Comarcas, são significativos e próximos. A proximidade do Núcleo com essas instituições têm ocorrido por meio da realização de estudos, tendo esses espaços como campo de pesquisa.

As parcerias nacionais e internacionais, com desenvolvimento de pesquisas, intercâmbio, participação em projetos, oficinas, bancas examinadoras, grupos de pesquisa, podem ser evidenciadas como significativas e próximas. Entretanto, com a Université du Quebec à Trois Rivierés, Canadá, o vínculo ainda é inexistente.

Ao se considerar o âmbito da América Latina, a Enfermagem brasileira tem atuação de cooperação em relação aos demais países. Entretanto, o estabelecimento de outros vínculos são necessários, para o desenvolvimento global da produção do conhecimento nos grupos de pesquisa em Enfermagem ${ }^{(22)}$. Para tanto deve-se considerar as redes sociais envolvidas ${ }^{(23-25)}$. A criação de redes formadoras de doutores em Enfermagem é mencionada como um modo de formar massa crítica competente para que, após a conclusão do doutorado, se torne multiplicadora desse tipo de formação em seus locais de origem ${ }^{(22)}$. As atividades relacionadas à produção de conhecimentos, por meio da pesquisa, caminham junto ao crescimento dos grupos de pesquisa que, no Brasil, tem sido proporcional ao crescimento da pós-graduação(2).

Deve ser estabelecida agenda que leve em consideração os desafios e demandas da academia na formação de pesquisadores, no consumo e na disseminação do conhecimento, no sentido de reduzir a distância entre a produção científica e a prática assistencial(22). Desse modo, para que o PROASE atinja plenamente o seu objetivo de promover a construção do conhecimento, no campo da educação e da saúde da criança e do adolescente, por meio da formação e qualificação de pesquisadores e recursos humanos, com ênfase na interdisciplinaridade, multiprofissionalidade e intersetorialidade, ensino de graduação, pós-graduação e 
trabalhos de extensão à comunidade, será necessário estabelecer agenda que contemple a complexidade contida nesse escopo.

Neste panorama, a prática da construção de conhecimento interdisciplinar exige do pesquisador uma postura diferenciada daquela que tradicionalmente é adotada. A necessidade de desenvolver novos paradigmas e métodos, essenciais para assegurar o rigor e a confiabilidade do conhecimento gerado, exige a renúncia de paradigmas e métodos já consolidados nos diversos campos disciplinares, bem como a constituição de equipes verdadeiramente multidisciplinares, o que se configura como um desafio a ser superado pelos Grupos de Pesquisa em Educação em Enfermagem brasileiros. Do mesmo modo, outro aspecto que merece destaque tratase da fragilidade da integração ensino-serviço dentro dos Grupos, manifestada pela prevalência de pesquisadores e técnicos que atuam exclusivamente na docência. Muitos desafios necessitam ser superados como a frágil interdisciplinaridade, o baixo fomento de bolsas para o avanço da área da Educação em Enfermagem e as significativas desigualdades no acesso e desenvolvimento de pesquisas nas diferentes regiões geográficas do país ${ }^{(3)}$.

\section{CONSIDERAÇÕES FINAIS}

O estudo mostrou que o PROASE atua em rede, estabelecendo vínculos com diferentes setores e instituições, caracteristicamente heterogêneos, apresentando tendência à abertura. Essa heterogeneidade se explica pela complexidade do objeto de estudo que tem interface com diversos setores e disciplinas. Outro aspecto, identificado em relação ao tamanho da rede, foi a avaliação como mediana, pelo número de instituições e setores com os quais o Núcleo se relaciona. Quanto à densidade, o mapa permite considerar predominância de vínculos significativos, caracterizando boa densidade.

Por outro lado, constata-se a fragilidade ou inexistência de articulação com parceiros que se situam nos extremos, em se tratando de distância geográfica, sendo os mais próximos os núcleos da EERP e os mais distantes, as instituições internacionais.

Ao se considerar o objetivo do PROASE de promover a construção do conhecimento no campo da Educação e Saúde da Criança e do Adolescente, por meio da formação e qualificação de pesquisadores e recursos humanos, ao se visualizar a configuração da rede em torno do PROASE, e partindo do entendimento de que os vínculos fortalecem a rede, acredita-se que oportunidades são perdidas no que se refere à realização do potencial do Núcleo e da rede como um todo, especialmente nas atividades de ensino, pesquisa e extensão, não deixando de repercutir na assistência às crianças e aos adolescentes.

Considerando tal contexto, o Núcleo ainda possui grandes desafios no sentido de se fortalecer a rede, por meio do aumento dos vínculos significativos, dessa forma fica o compromisso de intensificar parceria com grupos de pesquisa da EERP/USP; implementar parcerias internacionais para o desenvolvimento de pesquisas colaborativas e intensificar as parcerias com a Secretaria Municipal de Educação de Ribeirão Preto e escolas da rede municipal. Como também fortalecer outras parcerias com profissionais dos serviços de saúde, assistência social, educação e representantes das organizações da sociedade civil.

As recomendações aqui elencadas envolvem ações de médio e longo prazo, a serem implementadas pelo PROASE, num esforço coletivo dos seus membros, demandando, também, resposta das demais instituições.

Considera-se que o presente estudo contribui para a reflexão sobre a necessária resposta das universidades e especificamente dos grupos de pesquisa às necessidades da sociedade. Contudo, a discussão não se esgota, pois se entende serem necessários outros estudos, permitindo que novas luzes sejam lançadas sobre a realidade dos núcleos de pesquisa em Enfermagem.

2005 a 2007. Esc. Anna Nery [Internet] 2008 jun 12(2): 316-322. Disponivel em:

http://dx.doi.org/10.1590/S141481452008000200017.

3. Backes, VMS et. al. Grupos de Pesquisa de Educação em Enfermagem do Brasil. Rev. Esc. Enferm. USP, São Paulo,2012, Abril v.46,n.2. Disponível em: http://dx.doi.org/10.1590/S008062342012000200023.
1. Alexandre AMC, Maciel KF, Fernandes APP, Peres AM, Wolff LDG, Mantovani MF et al. Rede social de apoio no Brasil: grupos e linhas de pesquisa. Texto Contexto Enferm [Internet]. 2011 [acesso em: 29 mar 2013];20(2):241-6. Disponível em: http://dx.doi.org/10.1590/S0104-07072011000200004. 2. Lorenzini EA, Lanzoni GMM. Características dos grupos de pesquisa da enfermagem brasileira certificados pelo CNPq de

Rev. Eletr. Enf. [Internet]. 2013 jan/mar;15(1):172-81. Disponível em: http://dx.doi.org/10.5216/ree.v15i1.16597. doi: $10.5216 /$ ree.v15i1.16957. 
4. Barros, ALBL; Carneiro, CS e Santos, VB. A educação em saúde: um campo de atuação clínica e de pesquisa na enfermagem. Acta Paul. Enferm. [online]. 2011, vol.24, n.2 ISSN 0103-2100.

5. Gehlen, ST; Maldaner, OA e Delizoicov, D. Momentos pedagógicos e as etapas da situação de estudo: complementaridades e contribuições para a Educação em Ciências. Ciênc. Educ. (Bauru) [online]. 2012, vol.18, n.1 ISSN 1516-7313.

6. Alvarenga, AT; Vasconcellos, MP e Adorno, RCF. A contribuição das ciências sociais e humanas na pesquisa, no ensino e na formação em saúde. Saúde Soc. [online]. 2011, vol.20, n.1 ISSN 0104-1290.

7. Gomes, LB e Merhy, EE. Compreendendo a educação popular em saúde: um estudo na literatura brasileira. Cad. Saúde Pública [online]. 2011, vol.27, n.1 ISSN 0102-311X.

8. Ribeiro KSQS, Ampliando a atenção à saúde pela valorização das Redes sociais nas práticas de educação popular em saúde. Rev APS. jul-set 2008;11(3):235-48.

9. Conselho Nacional de Desenvolvimento Científico e Tecnológico CNPq. Grupos de Pesquisa: saiba mais. Disponível em: http://www.cnpq.br/gpesq/saibamais.htm/

10. Pereira MS, Ribeiro LCM, Mendonça KM, Tipple AFV, Souza ACS, Palos MAP et al. Grupo de Pesquisa em Enfermagem na Prevenção e Controle de Infecções: 20 anos de contribuições. Rev. Eletr. Enf. [Internet]. 2011 jan/mar;13(1):124-9. Disponível em: http://www.fen.ufg.br/revista/v13/n1/v13n1a14.htm 11. Meireles BHS, Erdmann AL. A questão das disciplinas e da interdisciplinaridade como processo educativo na área da saúde. Texto Contexto Enferm. 1999; 8(10): 149-65.

12. Ude W. Redes sociais: Possibilidade metodológica para uma prática inclusiva. In: Carvalho A et al.(org.). Políticas Públicas. 2002, Belo Horizonte: Proex/UFMG.

13. Amorim DS, Gattás MLB. Modelo de prática interdisciplinar em área na saúde. Revista Medicina Ribeirão Preto.

2007;40(1):82-4

14. Minayo MCS. O desafio do conhecimento: pesquisa qualitativa em saúde. 12th ed. São Paulo: Hucitec; 2010. 15. Felizardo Júnior LCF, Ude W. Enfrentamento à violência, configuração e redes sociais: possibilidades teóricometodológicas para a realização de intervenções. In: Santos GL, Felizardo Júnior LCF, Ude W. (orgs.) Escola, violência e redes sociais. Belo Horizonte. FAE/UFMG 2009.

16. Sluzky CE. A rede social na prática sistêmica. $3^{a}$ ed. São Paulo: Casa do Psicólogo; 2006.

17. Ude W. Enfrentamento da violência sexual infanto-juvenil e construção de redes sociais: produção e construção de redes sociais: produção de indicadores e possibilidades de intervenção. In: Cunha E, Silva EM, Giovanetti AC.

Enfrentamento à violência sexual Infanto-Juvenil: expansão do PAIR em Minas Gerais. Belo Horizonte: UFMG; 2008.

18. Silva LMP. A prevenção da violência sexual intrafamiliar contra crianças e adolescentes, sob a ótica dos membros do Poder Judiciário. [Tese] Ribeirão Preto:Universidade de São Paulo; 2010

19. Ludke M, André MED. A pesquisa em educação: abordagens qualitativas. São Paulo: EDU; 1986.

20. Ferriani MCF. A inserção do enfermeiro na saúde escolar: análise crítica de uma experiência. [Tese] Ribeirão Preto: Universidade de São Paulo; 1988.

21. Almeida MCP. Prefácio. In: Ferriani MGC. A inserção do enfermeiro na saúde escolar: análise crítica de uma experiência. São Paulo: Edusp; 1991.

22. Evangelista $\mathrm{Cl}$, Rubio TMA. Pesquisa em enfermagem nas Américas. Rev. Bras. Enferm. [Internet] 2010 [acesso em: 29 mar 2013];63(1):104-10. Disponível em:

http://dx.doi.org/10.1590/S003471672010000100017.
23. Rodrigues ML. Metodologia multidimensional em Ciências Humanas: um ensaio a partir do pensamento de Edgar Morin. In: Rodrigues, M.L.; Limena, C. M. M. (org.). Metodologias multidimensionais em Ciências Humanas. Brasília: Liber Livro Editora; 2006.

24. Sudbrack MFO. Redes sociais e os adolescentes: avaliação das redes sociais dos adolescentes. In: Universidade de Brasília. Curso de prevenção do uso drogas para educadores de escolas públicas. Brasília: UnB; 2006.

25. Vasconcelos ML. Avaliação das redes sociais da escola: uma estratégia de prevenção do uso de drogas. [Dissertação] Brasília: UnB; 2008.

Artigo recebido em 24/01/2012.

Aprovado para publicação em 14/08/2012.

Artigo publicado em 31/03/2013. 